\title{
Compact Embeddings for Spaces of Forward Rate Curves
}

\author{
Stefan Tappe \\ Institut für Mathematische Stochastik, Leibniz Universität Hannover, Welfengarten 1, 30167 Hannover, Germany \\ Correspondence should be addressed to Stefan Tappe; tappe@stochastik.uni-hannover.de \\ Received 19 June 2013; Accepted 2 September 2013 \\ Academic Editor: Alberto Parmeggiani \\ Copyright (C) 2013 Stefan Tappe. This is an open access article distributed under the Creative Commons Attribution License, which \\ permits unrestricted use, distribution, and reproduction in any medium, provided the original work is properly cited. \\ The goal of this paper is to prove a compact embedding result for spaces of forward rate curves. As a consequence of this result, we \\ show that any forward rate evolution can be approximated by a sequence of finite dimensional processes in the larger state space.
}

\section{Introduction}

The Heath-Jarrow-Morton-Musiela (HJMM) equation is a stochastic partial differential equation that models the evolution of forward rates in a market of zero coupon bonds; we refer to [1] for further details. It has been studied in a series of papers; see, for example, [2-5] and references therein. The state space, which contains the forward curves, is a separable Hilbert space $H$ consisting of functions $h: \mathbb{R}_{+} \rightarrow \mathbb{R}$. In practice, forward curves have the following features.

(i) The functions $h \in H$ become flat at the long end.

(ii) Consequently, the $\operatorname{limit}_{x \rightarrow \infty} h(x)$ exists.

The second property is taken into account by choosing the Hilbert space

$$
L_{\beta}^{2} \oplus \mathbb{R}
$$

where $L_{\beta}^{2}$ denotes the weighted Lebesgue space

$$
L_{\beta}^{2}:=L^{2}\left(\mathbb{R}_{+}, e^{\beta x} d x\right)
$$

for some constant $\beta>0$. Such spaces have been used, for example, in $[2,3]$. As flatness of a function is measured by its derivative, the first property is taken into account by choosing the space

$$
\begin{aligned}
& H_{\gamma} \\
& :=\left\{h: \mathbb{R}_{+} \longrightarrow \mathbb{R}: h \text { is absolutely continuous with }\|h\|_{\gamma}<\infty\right\},
\end{aligned}
$$

for some constant $\gamma>0$, where the norm is given by

$$
\|h\|_{\gamma}:=\left(|h(0)|^{2}+\int_{\mathbb{R}_{+}}\left|h^{\prime}(x)\right|^{2} e^{\gamma x} d x\right)^{1 / 2} .
$$

Such spaces have been introduced in [1] (even with more general weight functions) and further utilized, for example, in $[4,5]$. Our goal of this paper is to show that for all $\gamma>\beta>0$ we have the compact embedding

$$
H_{\gamma} \subset \subset L_{\beta}^{2} \oplus \mathbb{R}
$$

that is, the forward curve spaces used in [1] and forthcoming papers are contained in the forward curve spaces used in [2], and the embedding is even compact. Consequently, the embedding operator between these spaces can be approximated by a sequence of finite-rank operators, and hence, when considering the HJMM equation in the state space $H_{\gamma}$, applying these operators its solutions can be approximated by a sequence of finite dimensional processes in the larger state space $L_{\beta}^{2} \oplus \mathbb{R}$; we refer to Section 3 for further details.

The remainder of this paper is organized as follows. In Section 2, we provide the required preliminaries. In Section 3, we present the embedding result and its proof, and we outline the described approximation result concerning solutions of the HJMM equation.

\section{Preliminaries and Notation}

In this section, we provide the required preliminary results and some basic notation. Concerning the upcoming results 
about Sobolev spaces and Fourier transforms, we refer to any textbook about functional analysis, such as [6] or [7].

As noted in the introduction, for positive real numbers $\beta, \gamma>0$, the separable Hilbert spaces $L_{\beta}^{2} \oplus \mathbb{R}$ and $H_{\gamma}$ are given by (2) and (3), respectively. These spaces and the forthcoming Sobolev spaces will be regarded as spaces of complex-valued functions. For every $h \in H_{\gamma}$, the limit $h(\infty):=\lim _{x \rightarrow \infty} h(x)$ exists, and the subspace

$$
H_{\gamma}^{0}:=\left\{h \in H_{\gamma}: h(\infty)=0\right\}
$$

is a closed subspace of $H_{\gamma}$; see [1]. For an open set $\Omega \subset \mathbb{R}$, we denote by $W^{1}(\Omega)$ the Sobolev space

$$
W^{1}(\Omega):=\left\{f \in L^{2}(\Omega): f^{\prime} \in L^{2}(\Omega) \text { exists }\right\},
$$

which, equipped with the inner product

$$
\langle f, g\rangle_{W^{1}(\Omega)}=\langle f, g\rangle_{L^{2}(\Omega)}+\left\langle f^{\prime}, g^{\prime}\right\rangle_{L^{2}(\Omega)}
$$

is a separable Hilbert space. Here, derivatives are understood as weak derivatives.

For a function $h \in W^{1}((0, \infty))$, the extension $h \mathbb{1}_{(0, \infty)}$ : $\mathbb{R} \rightarrow \mathbb{C}$ in general, does not belong to $W^{1}(\mathbb{R})$. In the present situation, this technical problem can be resolved as follows. Let $h:(0, \infty) \rightarrow \mathbb{C}$ be a continuous function such that the limit $h(0):=\lim _{x \rightarrow 0} h(x)$ exists. Then, we define the reflection $h^{*}: \mathbb{R} \rightarrow \mathbb{C}$ as

$$
h^{*}(x):= \begin{cases}h(x), & \text { if } x \geq 0 \\ h(-x), & \text { if } x<0\end{cases}
$$

Lemma 1. The following statements are true.

(1) For each $h \in W^{1}((0, \infty))$, one has $h^{*} \in W^{1}(\mathbb{R})$.

(2) The mapping $W^{1}((0, \infty)) \rightarrow W^{1}(\mathbb{R}), h \mapsto h^{*}$ is a bounded linear operator.

(3) For each $h \in W^{1}((0, \infty))$, one has

$$
\begin{aligned}
\|h\|_{W^{1}((0, \infty))} & \leq\left\|h^{*}\right\|_{W^{1}(\mathbb{R})} \leq \sqrt{2}\|h\|_{W^{1}((0, \infty))}, \\
\|h\|_{L^{2}((0, \infty))} & \leq\left\|h^{*}\right\|_{L^{2}(\mathbb{R})} \leq \sqrt{2}\|h\|_{L^{2}((0, \infty))} .
\end{aligned}
$$

Proof. This follows from a straightforward calculation following the proof of [8, Theorem 8.6].

Lemma 2. Let $\gamma>\beta>0$ be arbitrary. Then, the following statements are true.

(1) One has $H_{\gamma}^{0} \subset H_{\beta}^{0}$, and

$$
\|h\|_{\beta} \leq\|h\|_{\gamma} \quad \forall h \in H_{\gamma}^{0} .
$$

(2) One has $H_{\gamma}^{0} \subset L_{\beta}^{2}$, and there is a constant $C_{1}=C_{1}(\beta$, $\gamma)>0$ such that

$$
\|h\|_{L_{\beta}^{2}} \leq C_{1}\|h\|_{\gamma} \quad \forall h \in H_{\gamma}^{0}
$$

(3) For each $h \in H_{\gamma}^{0}$, one has

$$
\left.h e^{(\beta / 2) \bullet}\right|_{(0, \infty)} \in W^{1}((0, \infty)), \quad\left(\left.h e^{(\beta / 2) \bullet}\right|_{(0, \infty)}\right)^{*} \in W^{1}(\mathbb{R}),
$$

and there is a constant $C_{2}=C_{2}(\beta, \gamma)>0$ such that

$$
\left\|\left(\left.h e^{(\beta / 2) \cdot}\right|_{(0, \infty)}\right)^{*}\right\|_{W^{1}(\mathbb{R})} \leq C_{2}\|h\|_{\gamma} \quad \forall h \in H_{\gamma}^{0} \text {. }
$$

Proof. The first statement is a direct consequence of the representation of the norm on $H_{\gamma}^{0}$ given by (4). Let $h \in H_{\gamma}^{0}$ be arbitrary. By the Cauchy-Schwarz inequality, we obtain

$$
\begin{aligned}
\|h\|_{L_{\beta}^{2}}^{2} & =\int_{\mathbb{R}_{+}}|h(x)|^{2} e^{\beta x} d x \\
& =\int_{\mathbb{R}_{+}}\left(\int_{x}^{\infty} h^{\prime}(\eta) e^{(\gamma / 2) \eta} e^{-(\gamma / 2) \eta} d \eta\right)^{2} e^{\beta x} d x \\
& \leq \int_{\mathbb{R}_{+}}\left(\int_{x}^{\infty}\left|h^{\prime}(\eta)\right|^{2} e^{\gamma \eta} d \eta\right)\left(\int_{x}^{\infty} e^{-\gamma \eta} d \eta\right) e^{\beta x} d x \\
& \leq \int_{\mathbb{R}_{+}}\left(\int_{\mathbb{R}_{+}}\left|h^{\prime}(\eta)\right|^{2} e^{\gamma \eta} d \eta\right) \frac{1}{\gamma} e^{-\gamma x} e^{\beta x} d x \\
& \leq \frac{1}{\gamma}\left(\int_{\mathbb{R}_{+}} e^{-(\gamma-\beta) x} d x\right)\|h\|_{\gamma}^{2}=\frac{1}{\gamma(\gamma-\beta)}\|h\|_{\gamma}^{2},
\end{aligned}
$$

proving the second statement. Furthermore, by (12) we have

$$
\begin{aligned}
& \left\|\left.h e^{(\beta / 2) \cdot}\right|_{(0, \infty)}\right\|_{L^{2}((0, \infty))}^{2} \\
& \quad=\int_{\mathbb{R}_{+}}\left|h(x) e^{(\beta / 2) x}\right|^{2} d x=\int_{\mathbb{R}_{+}}|h(x)|^{2} e^{\beta x} d x \\
& =\|h\|_{L_{\beta}^{2}}^{2} \leq C_{1}^{2}\|h\|_{\gamma}^{2},
\end{aligned}
$$

and by estimates (11), (12), we obtain

$$
\begin{aligned}
& \|\left(\frac{d}{d x}\right)\left(h e^{\left.\left.(\beta / 2) \cdot\right|_{(0, \infty)}\right)} \|_{L^{2}((0, \infty))}^{2}\right. \\
& =\int_{\mathbb{R}_{+}}\left|\frac{d}{d x}\left(h(x) e^{(\beta / 2) x}\right)\right|^{2} d x \\
& \quad=\int_{\mathbb{R}_{+}}\left|h^{\prime}(x) e^{(\beta / 2) x}+\frac{\beta}{2} h(x) e^{(\beta / 2) x}\right|^{2} d x \\
& \leq 2\left(\int_{\mathbb{R}_{+}}\left|h^{\prime}(x)\right|^{2} e^{\beta x} d x+\frac{\beta^{2}}{4} \int_{\mathbb{R}_{+}}|h(x)|^{2} e^{\beta x} d x\right) \\
& \leq 2\|h\|_{\beta}^{2}+\frac{\beta^{2}}{2}\|h\|_{L_{\beta}^{2}} \leq\left(2+\frac{\beta^{2} C_{1}^{2}}{2}\right)\|h\|_{\gamma}^{2},
\end{aligned}
$$

which, together with Lemma 1, concludes the proof.

For $h \in L^{1}(\mathbb{R})$, the Fourier transform $\mathscr{F} h: \mathbb{R} \rightarrow \mathbb{C}$ is defined as

$$
(\mathscr{F} h)(\xi):=\frac{1}{\sqrt{2 \pi}} \int_{\mathbb{R}} h(x) e^{-i \xi x} d x, \quad \xi \in \mathbb{R} .
$$


Recall that $C_{0}(\mathbb{R})$ denotes the space of all continuous functions vanishing at infinity, which, equipped with the supremum norm, is a Banach space. We have the following result.

Lemma 3. The Fourier transform $\mathscr{F}: L^{1}(\mathbb{R}) \rightarrow C_{0}(\mathbb{R})$ is a continuous linear operator with $\|\mathscr{F}\| \leq 1 / \sqrt{2 \pi}$.

Lemma 4. Let $\gamma>\beta>0$ be arbitrary. Then, the following statements are true.

(1) For each $h \in H_{\gamma}^{0}$, one has $\left(\left.h e^{(\beta / 2) \cdot}\right|_{(0, \infty)}\right)^{*} \in L^{1}(\mathbb{R})$, and there is a constant $C_{3}=C_{3}(\beta, \gamma)>0$ such that

$$
\left\|\left(\left.h e^{(\beta / 2) \cdot}\right|_{(0, \infty)}\right)^{*}\right\|_{L^{1}(\mathbb{R})} \leq C_{3}\|h\|_{\gamma} \quad \forall h \in H_{\gamma}^{0}
$$

(2) For each $\xi \in \mathbb{R}$, the mapping

$$
H_{\gamma}^{0} \longrightarrow \mathbb{R}, \quad h \longmapsto \mathscr{F}\left(\left.h e^{(\beta / 2) \cdot}\right|_{(0, \infty)}\right)^{*}(\xi)
$$

is a continuous linear functional.

Proof. We set $\delta:=(1 / 2)(\beta+\gamma) \in(\beta, \gamma)$. Let $h \in H_{\gamma}^{0}$ be arbitrary. By the Cauchy-Schwarz inequality and Lemma 2, we have

$$
\begin{aligned}
& \left\|\left(\left.h e^{(\beta / 2) \bullet}\right|_{(0, \infty)}\right)^{*}\right\|_{L^{1}(\mathbb{R})} \\
& \quad=2\left\|h e^{(\beta / 2) \cdot}\right\|_{L^{1}\left(\mathbb{R}_{+}\right)}=2 \int_{\mathbb{R}_{+}}\left|h(x) e^{(\beta / 2) x}\right| d x \\
& =2 \int_{\mathbb{R}_{+}}|h(x)| e^{(\delta / 2) x} e^{-((\delta-\beta) / 2) x} d x \\
& \leq 2\left(\int_{\mathbb{R}_{+}}|h(x)|^{2} e^{\delta x} d x\right)^{1 / 2}\left(\int_{\mathbb{R}_{+}} e^{-(\delta-\beta) x} d x\right)^{1 / 2} \\
& =2 \sqrt{\frac{1}{\delta-\beta}}\|h\|_{L_{\delta}^{2}} \leq 2 C_{1}(\delta, \gamma) \sqrt{\frac{1}{\delta-\beta}}\|h\|_{\gamma},
\end{aligned}
$$

showing the first statement. Moreover, we have

$$
\begin{aligned}
\left\|e^{((\beta / 2)-\delta) \bullet}\right\|_{L_{\delta}^{2}}^{2} & =\int_{\mathbb{R}_{+}} e^{2((\beta / 2)-\delta) x} e^{\delta x} d x \\
& =\int_{\mathbb{R}_{+}} e^{-(\delta-\beta) x} d x=\frac{1}{\delta-\beta},
\end{aligned}
$$

showing that $e^{((\beta / 2)-\delta) \bullet} \in L_{\delta}^{2}$. Let $h \in H_{\gamma}^{0}$ and $\xi \in \mathbb{R}$ be arbitrary. By Lemma 2, we have $h \in L_{\delta}^{2}$, and hence

$$
\begin{aligned}
& \mathscr{F}\left(\left.h e^{(\beta / 2) \bullet}\right|_{(0, \infty)}\right)^{*}(\xi) \\
& =\frac{1}{\sqrt{2 \pi}}\left(\int_{0}^{\infty} h(x) e^{(\beta / 2) x} e^{-i \xi x} d x\right. \\
& \left.\quad+\int_{-\infty}^{0} h(-x) e^{-(\beta / 2) x} e^{-i \xi x} d x\right) \\
& =\frac{1}{\sqrt{2 \pi}}\left(\int_{0}^{\infty} h(x) e^{(\beta / 2) x} e^{-i \xi x} d x+\int_{0}^{\infty} h(x) e^{(\beta / 2) x} e^{i \xi x} d x\right) \\
& =\frac{1}{\sqrt{2 \pi}}\left\langle h, e^{((\beta / 2)-\delta) \bullet}\left(e^{-i \xi \bullet}+e^{i \xi \bullet}\right)\right\rangle_{L_{\delta}^{2}},
\end{aligned}
$$

proving the second statement.

We can also define the Fourier transform on $L^{2}(\mathbb{R})$ such that $\mathscr{F}: L^{2}(\mathbb{R}) \rightarrow L^{2}(\mathbb{R})$ is a bijection, and we have the Plancherel isometry

$$
\langle\mathscr{F} f, \mathscr{F} g\rangle_{L^{2}(\mathbb{R})}=\langle f, g\rangle_{L^{2}(\mathbb{R})} \quad \forall f, g \in L^{2}(\mathbb{R}) .
$$

Moreover, the two just reviewed definitions of the Fourier transform coincide on $L^{1}(\mathbb{R}) \cap L^{2}(\mathbb{R})$. For each $h \in W^{1}(\mathbb{R})$, we have

$$
\left(\mathscr{F} h^{\prime}\right)(\xi)=i \xi(\mathscr{F} h)(\xi), \quad \xi \in \mathbb{R} .
$$

Lemma 5. For every $h \in W^{1}(\mathbb{R})$, one has

$$
\|\cdot \mathscr{F} h\|_{L^{2}(\mathbb{R})} \leq\|h\|_{W^{1}(\mathbb{R})} .
$$

Proof. Let $h \in W^{1}(\mathbb{R})$ be arbitrary. By identity (25) and the Plancherel isometry (24), we have

$$
\|\cdot \mathscr{F} h\|_{L^{2}(\mathbb{R})}=\left\|\mathscr{F} h^{\prime}\right\|_{L^{2}(\mathbb{R})}=\left\|h^{\prime}\right\|_{L^{2}(\mathbb{R})} \leq\|h\|_{W^{1}(\mathbb{R})}
$$

finishing the proof.

\section{The Embedding Result and Its Proof}

In this section, we present the compact embedding result and its proof.

Theorem 6. For all $\gamma>\beta>0$, one has the compact embedding

$$
H_{\gamma} \subset \subset L_{\beta}^{2} \oplus \mathbb{R}
$$

Proof. Noting that $H_{\gamma} \cong H_{\gamma}^{0} \oplus \mathbb{R}$, it suffices to prove the compact embedding $H_{\gamma}^{0} \subset \subset L_{\beta}^{2}$. Let $\left(h_{j}\right)_{j \in \mathbb{N}} \subset H_{\gamma}^{0}$ be a bounded sequence. Then, there exists a subsequence which converges weakly in $H_{\gamma}^{0}$. Without loss of generality, we may assume that the original sequence $\left(h_{j}\right)_{j \in \mathbb{N}}$ converges weakly 
in $H_{\gamma}^{0}$. We will prove that $\left(h_{j}\right)_{j \in \mathbb{N}}$ is a Cauchy sequence in $L_{\beta}^{2}$. According to Lemma 2 , the sequence $\left(g_{j}\right)_{j \in \mathbb{N}}$ given by

$$
g_{j}:=\left(\left.h_{j} e^{(\beta / 2) \bullet}\right|_{(0, \infty)}\right)^{*}, \quad j \in \mathbb{N}
$$

is a bounded sequence in $W^{1}(\mathbb{R})$. By Lemma 1 and the Plancherel isometry (24), for all $j, k \in \mathbb{N}$, we get

$$
\begin{aligned}
\left\|h_{k}-h_{j}\right\|_{L_{\beta}^{2}}^{2} & =\| h_{k} e^{(\beta / 2) \bullet}-h_{j} e^{(\beta / 2) \cdot \|_{L^{2}\left(\mathbb{R}_{+}\right)}^{2}} \\
& \leq\left\|g_{k}-g_{j}\right\|_{L^{2}(\mathbb{R})}^{2}=\left\|\mathscr{F} g_{k}-\mathscr{F} g_{j}\right\|_{L^{2}(\mathbb{R})}^{2} \\
& =\int_{\mathbb{R}}\left|\left(\mathscr{F} g_{k}\right)(x)-\left(\mathscr{F} g_{j}\right)(x)\right|^{2} d x .
\end{aligned}
$$

Thus, for every $R>0$ we obtain the estimate

$$
\begin{aligned}
\left\|h_{k}-h_{j}\right\|_{L_{\beta}^{2}}^{2} \leq & \int_{\{|x| \leq R\}}\left|\left(\mathscr{F} g_{k}\right)(x)-\mathscr{F}\left(g_{j}\right)(x)\right|^{2} d x \\
& +\int_{\{|x|>R\}}\left|\left(\mathscr{F} g_{k}\right)(x)-\mathscr{F}\left(g_{j}\right)(x)\right|^{2} d x .
\end{aligned}
$$

By Lemma 5 , the sequence $\left(\bullet \mathscr{F} g_{j}\right)_{j \in \mathbb{N}}$ is bounded in $L^{2}(\mathbb{R})$. Therefore, for an arbitrary $\epsilon>0$ there exists a real number $R>0$ such that

$$
\begin{array}{r}
\int_{\{|x|>R\}}\left|\left(\mathscr{F} g_{k}\right)(x)-\left(\mathscr{F} g_{j}\right)(x)\right|^{2} d x \\
\leq \frac{1}{R^{2}} \int_{\{|x|>R\}}|x|^{2}\left|\left(\mathscr{F} g_{k}\right)(x)-\left(\mathscr{F} g_{j}\right)(x)\right|^{2} d x<\epsilon \\
\forall j, k \in \mathbb{N} .
\end{array}
$$

By Lemma 4 , for each $\xi \in \mathbb{R}$ the mapping

$$
H_{\gamma}^{0} \longrightarrow \mathbb{R}, \quad h \longmapsto \mathscr{F}\left(\left.h e^{(\beta / 2) \bullet}\right|_{(0, \infty)}\right)^{*}(\xi)
$$

is a continuous linear functional. Consequently, since $\left(h_{j}\right)_{j \in \mathbb{N}}$ converges weakly in $H_{\gamma}^{0}$, for each $\xi \in \mathbb{R}$, the real-valued sequence $\left(\left(\mathscr{F} g_{j}\right)(\xi)\right)_{j \in \mathbb{N}}$ is convergent. Moreover, by Lemmas 3 and 4 , for all $h \in H_{\gamma}^{0}$, we have the estimate

$$
\begin{aligned}
& \left\|\mathscr{F}\left(\left(\left.h e^{(\beta / 2) \cdot}\right|_{(0, \infty)}\right)^{*}\right)\right\|_{C_{0}(\mathbb{R})} \\
& \quad \leq \frac{1}{\sqrt{2 \pi}}\left\|\left(\left.h e^{(\beta / 2) \bullet}\right|_{(0, \infty)}\right)^{*}\right\|_{L^{1}(\mathbb{R})} \leq \frac{C_{3}}{\sqrt{2 \pi}}\|h\|_{\gamma} .
\end{aligned}
$$

Therefore, the sequence $\left(\mathscr{F} g_{j}\right)_{j \in \mathbb{N}}$ is bounded in $C_{0}(\mathbb{R})$. Using Lebesgue's dominated convergence theorem, we deduce that

$$
\int_{\{|x| \leq R\}}\left|\left(\mathscr{F} g_{k}\right)(x)-\left(\mathscr{F} g_{j}\right)(x)\right|^{2} d x \longrightarrow 0 \text { for } j, k \longrightarrow \infty \text {. }
$$

Combining (31) together with (32) and (35) shows that $\left(h_{j}\right)_{j \in \mathbb{N}}$ is a Cauchy sequence in $L_{\beta}^{2}$, completing the proof.
Remark 7. Note that the proof of Theorem 6 has certain analogies to the proof of the classical Rellich embedding theorem (see, e.g., [7, Theorem V.2.13]), which states the compact embedding $H_{0}^{1}(\Omega) \subset \subset L^{2}(\Omega)$ for an open, bounded subset $\Omega \subset \mathbb{R}^{n}$. Here, $H_{0}^{1}(\Omega)$ denotes the Sobolev space $H_{0}^{1}(\Omega)=\overline{\mathscr{D}(\Omega)}$, where $\mathscr{D}(\Omega)$ is the space of all $C^{\infty}$-functions on $\Omega$ with compact support, and where the closure is taken with respect to the topology induced by the inner product $\langle\cdot, \cdot\rangle_{W^{1}}$. Let us briefly describe the analogies and differences between the two results as follows.

(i) In the classical Rellich embedding theorem, the domain $\Omega$ is assumed to be bounded, whereas in Theorem 6 we have $\Omega=\mathbb{R}_{+}$. Moreover, we consider weighted function spaces with weight functions of the type $w(x)=e^{\beta x}$ for some constant $\beta>0$. This requires a careful analysis of the results regarding Fourier transforms which we have adapted to the present situation; see Lemma 4.

(ii) $H_{\gamma}$ and $H_{0}^{1}(\Omega)$ are different kinds of spaces. While the norm on $H_{0}^{1}(\Omega)$ given by $(8)$ involves the $L^{2}$-norms of a function $h$ and its derivative $h^{\prime}$, the norm (4) on $H_{\gamma}$ only involves the $L^{2}$-norm of the derivative $h^{\prime}$ and a point evaluation. Therefore, the embedding $H_{0}^{1}(\Omega) \subset$ $L^{2}(\Omega)$ follows right away, whereas we require the assumption $\beta<\gamma$ for the embedding $H_{\gamma}^{0} \subset L_{\beta}^{2}$; see Lemma 2.

(iii) The classical Rellich embedding theorem does not need to be true with $H_{0}^{1}(\Omega)$ being replaced by $W^{1}(\Omega)$. The reason behind this is that, in general, it is not possible to extend a function $h \in W^{1}(\Omega)$ to a function $\widetilde{h} \in W^{1}\left(\mathbb{R}^{n}\right)$, which, however, is crucial in order to apply the results about Fourier transforms. Usually, one assumes that $\Omega$ satisfies a so-called cone condition; see, for example, [9] for further details. In our situation, we have to ensure that every function $h \in H_{\gamma}^{0}$ can be extended to a function $\widetilde{h} \in W^{1}(\mathbb{R})$, and this is provided by Lemma 2 .

For the rest of this section, we will describe the announced application regarding the approximation of solutions to semilinear stochastic partial differential equations (SPDEs), which in particular applies to the modeling of interest rates. Consider a SPDE of the form

$$
\begin{gathered}
d r_{t}=\left(A r_{t}+\alpha\left(t, r_{t}\right)\right) d t+\sigma\left(t, r_{t}\right) d W_{t} \\
+\int_{E} \gamma\left(t, r_{t-}, \xi\right)(\mathfrak{p}(d t, d \xi)-v(d \xi) d t) \\
r_{0}=h_{0}
\end{gathered}
$$

on some separable Hilbert space $H_{1}$ with $A$ denoting the generator of some strongly continuous semigroup on $H_{1}$, driven by a Wiener process $W$ and a homogeneous Poisson random measure $\mathfrak{p}$ with compensator $d t \otimes \nu(d \xi)$ on some mark space $E$. We assume that the standard Lipschitz and linear growth conditions are satisfied which ensure for each 
initial condition $h_{0} \in H_{1}$ the existence of a unique weak solution $r$ to (36); that is, for each $\zeta \in \mathscr{D}\left(A^{*}\right)$, we have almost surely

$$
\begin{aligned}
&\left\langle\zeta, r_{t}\right\rangle=\left\langle\zeta, h_{0}\right\rangle_{H_{1}}+\int_{0}^{t}\left(\left\langle A^{*} \zeta, r_{s}\right\rangle_{H_{1}}+\left\langle\zeta, \alpha\left(s, r_{s}\right)\right\rangle_{H_{1}}\right) d s \\
&+\int_{0}^{t}\left\langle\zeta, \sigma\left(s, r_{s}\right)\right\rangle_{H_{1}} d W_{s} \\
&+\int_{0}^{t} \int_{E}\left\langle\zeta, \gamma\left(s, r_{s-}, \xi\right)\right\rangle_{H_{1}}(\mathfrak{p}(d s, d \xi)-\nu(d \xi) d s) \\
& \forall t \geq 0 ;
\end{aligned}
$$

see, for example, [10] for further details. Let $H_{2}$ be a larger separable Hilbert space with compact embedding $\mathrm{H}_{1} \subset \subset \mathrm{H}_{2}$. By virtue of Theorem 6, this is in particular satisfied for the forward curve spaces $H_{1}=H_{\gamma}$ and $H_{2}=L_{\beta}^{2} \oplus \mathbb{R}$ for $\gamma>$ $\beta>0$. If, furthermore, $A=d / d x$ is the differential operator, which is generated by the translation semigroup $\left(S_{t}\right)_{t \geq 0}$ given by $S_{t} h=h(t+\bullet)$, and $\alpha=\alpha_{\mathrm{HJM}}$ is given by the so-called HJM drift condition

$\alpha_{\mathrm{HJM}}(t, h)$

$$
\begin{aligned}
= & \sum_{j} \sigma^{j}(t, h) \int_{0}^{\bullet} \sigma^{j}(t, h)(\eta) d \eta \\
& -\int_{E} \gamma(t, h, \xi)\left[\exp \left(-\int_{0}^{\bullet} \gamma(t, h, \xi)(\eta) d \eta\right)-1\right] \nu(d \xi),
\end{aligned}
$$

then the SPDE (36), which in this case becomes the mentioned HJMM equation, describes the evolution of interest rates in an arbitrage free bond market; we refer to [5] for further details.

By virtue of the compact embedding $\mathrm{H}_{1} \subset \subset \mathrm{H}_{2}$, there exist orthonormal systems $\left(e_{k}\right)_{k \in \mathbb{N}}$ of $H_{1}$ and $\left(f_{k}\right)_{k \in \mathbb{N}}$ of $H_{2}$, and a decreasing sequence $\left(s_{k}\right)_{k \in \mathbb{N}} \subset \mathbb{R}_{+}$with $s_{k} \rightarrow 0$ such that

$$
h=\sum_{k=1}^{\infty} s_{k}\left\langle h, e_{k}\right\rangle_{H_{1}} f_{k} \quad \forall h \in H_{1} ;
$$

see, for example, [7, Theorem VI.3.6]. The numbers $s_{k}$ are the singular numbers of the identity operator Id $: H_{1} \rightarrow H_{2}$. Defining the sequence $\left(T_{n}\right)_{n \in \mathbb{N}}$ of finite-rank operators

$$
T_{n}: H_{1} \longrightarrow F_{n}, \quad T_{n} h:=\sum_{k=1}^{n} s_{k}\left\langle h, e_{k}\right\rangle_{H_{1}} f_{k},
$$

where $F_{n}:=\left\langle f_{1}, \ldots, f_{n}\right\rangle$, we even have $T_{n} \rightarrow$ Id with respect to the operator norm

$$
\|T\|:=\sup _{\|h\|_{H_{1}} \leq 1}\|T h\|_{H_{2}}
$$

see, for example, [7, Corollary VI.3.7]. Consequently, denoting by $r$ the weak solution to the SPDE (36) for some initial condition $h_{0} \in H_{1}$, the sequence $\left(T_{n}(r)\right)_{n \in \mathbb{N}}$ is a sequence of $F_{n}$-valued stochastic processes, and we have almost surely

$$
\left\|T_{n}\left(r_{t}\right)-r_{t}\right\|_{H_{2}} \leq\left\|T_{n}-\mathrm{Id}\right\|\left\|r_{t}\right\|_{H_{1}} \longrightarrow 0 \quad \forall t \geq 0
$$

showing that the weak solution $r$-when considered on the larger state space $\mathrm{H}_{2}$ - can be approximated by the sequence of finite dimensional processes $\left(T_{n}(r)\right)_{n \in \mathbb{N}}$ with distance between $T_{n}(r)$ and $r$ estimated in terms of the operator norm $\left\|T_{n}-\mathrm{Id}\right\|$, as shown in (42). However, the sequence $\left(T_{n}(r)\right)_{n \in \mathbb{N}}$ does not need to be a sequence of Itô processes. This issue is addressed by the following result.

Proposition 8. Let $\left(\epsilon_{n}\right)_{n \in \mathbb{N}} \subset(0, \infty)$ be an arbitrary decreasing sequence with $\epsilon_{n} \rightarrow 0$. Then, for every initial condition $h_{0} \in H_{1}$, there exists a sequence $\left(r^{(n)}\right)_{n \in \mathbb{N}}$ of $F_{n}$-valued Itô processes such that almost surely

$$
\left\|r_{t}^{(n)}-r_{t}\right\|_{H_{2}} \leq\left(\left\|T_{n}-\mathrm{Id}\right\|+\epsilon_{n}\right)\left\|r_{t}\right\|_{H_{1}} \longrightarrow 0 \quad \forall t \geq 0
$$

where $r$ denotes the weak solution to (36).

Proof. According to [6, Theorems 13.35.c and 13.12], the domain $\mathscr{D}\left(A^{*}\right)$ is dense in $H_{1}$. Therefore, for each $n \in \mathbb{N}$, there exist elements $\zeta_{1}^{(n)}, \ldots, \zeta_{n}^{(n)} \in \mathscr{D}\left(A^{*}\right)$ such that

$$
\left\|\zeta_{k}^{(n)}-e_{k}\right\|_{H_{1}}<\frac{\epsilon_{n}}{2^{k} \cdot s_{k}} \quad \forall k=1, \ldots, n
$$

where we use the convention $x / 0:=\infty$ for $x>0$. We define the sequence $\left(S_{n}\right)_{n \in \mathbb{N}}$ of finite-rank operators as

$$
S_{n}: H_{1} \longrightarrow F_{n}, \quad S_{n} h:=\sum_{k=1}^{n} s_{k}\left\langle h, \zeta_{k}^{(n)}\right\rangle_{H_{1}} f_{k} .
$$

By the geometric series, for all $n \in \mathbb{N}$, we have

$$
\begin{aligned}
\left\|S_{n}-\mathrm{Id}\right\| & \leq\left\|S_{n}-T_{n}\right\|+\left\|T_{n}-\mathrm{Id}\right\| \\
& \leq \sum_{k=1}^{n} s_{k}\left\|\left\langle\bullet, \zeta_{k}^{(n)}-e_{k}\right\rangle_{H_{1}}\right\|+\left\|T_{n}-\mathrm{Id}\right\| \\
& \leq \epsilon_{n} \sum_{k=1}^{n} \frac{1}{2^{k}}+\left\|T_{n}-\mathrm{Id}\right\| \leq \epsilon_{n}+\left\|T_{n}-\mathrm{Id}\right\| .
\end{aligned}
$$

For each $n \in \mathbb{N}$, let $r^{(n)}$ be the $F_{n}$-valued Itô process

$$
\begin{aligned}
r_{t}^{(n)}= & h_{0}^{(n)}+\int_{0}^{t} \alpha_{s}^{(n)} d s+\int_{0}^{t} \sigma_{s}^{(n)} d W_{s} \\
& +\int_{0}^{t} \int_{E} \delta_{s}^{(n)}(\xi)(\mathfrak{p}(d s, d \xi)-v(d \xi, d s)),
\end{aligned}
$$


with parameters given by

$$
\begin{aligned}
h_{0}^{(n)} & =\sum_{k=1}^{n} s_{k}\left\langle\zeta_{k}^{(n)}, h_{0}\right\rangle_{H_{1}} f_{k} \\
\alpha_{t}^{(n)} & =\sum_{k=1}^{n} s_{k}\left(\left\langle A^{*} \zeta_{k}^{(n)}, r_{t}\right\rangle_{H_{1}}+\left\langle\zeta_{k}^{(n)}, \alpha\left(t, r_{t}\right)\right\rangle_{H_{1}}\right) f_{k} \\
\sigma_{t}^{(n)} & =\sum_{k=1}^{n} s_{k}\left\langle\zeta_{k}^{(n)}, \sigma\left(t, r_{t}\right)\right\rangle_{H_{1}} f_{k} \\
\delta_{t}^{(n)}(\xi) & =\sum_{k=1}^{n} s_{k}\left\langle\zeta_{k}^{(n)}, \delta\left(t, r_{t-}, \xi\right)\right\rangle_{H_{1}} f_{k} .
\end{aligned}
$$

Since $r$ is a weak solution to (36), we obtain almost surely

$$
\begin{array}{rl}
S_{n}\left(r_{t}\right)= & \sum_{k=1}^{n} s_{k}\left\langle\zeta_{k}^{(n)}, r_{t}\right\rangle_{H_{1}} f_{k} \\
= & \sum_{k=1}^{n} s_{k}\left(\left\langle\zeta_{k}^{(n)}, h_{0}\right\rangle_{H_{1}}\right. \\
& \quad \int_{0}^{t}\left(\left\langle A^{*} \zeta_{k}^{(n)}, r_{s}\right\rangle_{H_{1}}+\left\langle\zeta_{k}^{(n)}, \alpha\left(s, r_{s}\right)\right\rangle_{H_{1}}\right) d s \\
& +\int_{0}^{t}\left\langle\zeta_{k}^{(n)}, \sigma\left(s, r_{s}\right)\right\rangle_{H_{1}} d W_{s} \\
& +\int_{0}^{t} \int_{E}\left\langle\zeta_{k}^{(n)}, \delta\left(s, r_{s-}, \xi\right)\right\rangle_{H_{1}} \\
& \times(\mathfrak{p}(d s, d \xi)-\nu(d \xi, d s)) f_{k} \\
= & h_{0}^{(n)}+\int_{0}^{t} \alpha_{s}^{(n)} d s+\int_{0}^{t} \sigma_{s}^{(n)} d W_{s} \\
= & r_{t}^{(n)}(\xi)(\mathfrak{p}(d s, d \xi)-v(d \xi, d s)) \\
\forall t & 0,
\end{array}
$$

which finishes the proof.

We will conclude this section with further consequences regarding the speed of convergence of the approximations $\left(r^{(n)}\right)_{n \in \mathbb{N}}$ provided by Proposition 8 . Let $h_{0} \in H_{1}$ be an arbitrary initial condition and denote by $r$ the weak solution to (36). Furthermore, let $T>0$ be a finite time horizon. Since

$$
\mathbb{E}\left[\sup _{t \in[0, T]}\left\|r_{t}\right\|_{H_{1}}^{2}\right]<\infty
$$

see, for example, [10, Corollary 10.3], by (43) there exists a constant $K>0$ such that

$$
\mathbb{E}\left[\sup _{t \in[0, T]}\left\|r_{t}^{(n)}-r_{t}\right\|_{H_{2}}^{2}\right]^{1 / 2} \leq K\left(\left\|T_{n}-\mathrm{Id}\right\|+\epsilon_{n}\right) \longrightarrow 0,
$$

providing a uniform estimate for the distance of $r^{(n)}$ and $r$ in the mean-square sense. Moreover, considering the pure diffusion case

$$
\begin{gathered}
d r_{t}=\left(A r_{t}+\alpha\left(t, r_{t}\right)\right) d t+\sigma\left(t, r_{t}\right) d W_{t} \\
r_{0}=h_{0},
\end{gathered}
$$

the sample paths of $r$ are continuous; for every constant $K>$ $\left\|h_{0}\right\|_{H_{1}}$ the stopping time

$$
\tau:=\inf \left\{t \geq 0:\left\|r_{t}\right\| \geq K\right\}
$$

is strictly positive, and by (43) for the stopped processes we obtain almost surely

$$
\sup _{t \in \mathbb{R}_{+}}\left\|r_{t \wedge \tau}^{(n)}-r_{t \wedge \tau}\right\|_{H_{2}} \leq K\left(\left\|T_{n}-\mathrm{Id}\right\|+\epsilon_{n}\right) \longrightarrow 0 ;
$$

that is, locally the solution $r$ stays in a bounded subset of $H_{\gamma}$ and we obtain the uniform convergence (54).

\section{Acknowledgment}

The author is grateful to an anonymous referee for valuable comments and suggestions.

\section{References}

[1] D. Filipović, Consistency Problems for Heath-Jarrow-Morton Interest Rate Models, vol. 1760 of Lecture Notes in Mathematics, Springer, Berlin, Germany, 2001.

[2] A. Rusinek, "Mean reversion for HJMM forward rate models," Advances in Applied Probability, vol. 42, no. 2, pp. 371-391, 2010.

[3] M. Barski and J. Zabczyk, "Heath-Jarrow-Morton-Musiela equation with Lévy perturbation," Journal of Differential Equations, vol. 253, no. 9, pp. 2657-2697, 2012.

[4] D. Filipović and S. Tappe, "Existence of Lévy term structure models," Finance and Stochastics, vol. 12, no. 1, pp. 83-115, 2008.

[5] D. Filipović, S. Tappe, and J. Teichmann, "Term structure models driven by Wiener processes and Poisson measures: existence and positivity," SIAM Journal on Financial Mathematics, vol. 1, no. 1, pp. 523-554, 2010.

[6] W. Rudin, Functional Analysis, International Series in Pure and Applied Mathematics, McGraw-Hill, New York, NY, USA, 2nd edition, 1991.

[7] D. Werner, Funktionalanalysis, Springer, Berlin, Germany, 2007.

[8] H. Brezis, Functional Analysis, Sobolev Spaces and Partial Differential Equations, Universitext, Springer, New York, NY, USA, 2011.

[9] R. A. Adams and J. J. F. Fournier, Sobolev Spaces, vol. 140 of Pure and Applied Mathematics, Elsevier/Academic Press, Amsterdam, The Netherlands, 2nd edition, 2003.

[10] D. Filipović, S. Tappe, and J. Teichmann, "Jump-diffusions in Hilbert spaces: existence, stability and numerics," Stochastics, vol. 82, no. 5, pp. 475-520, 2010. 


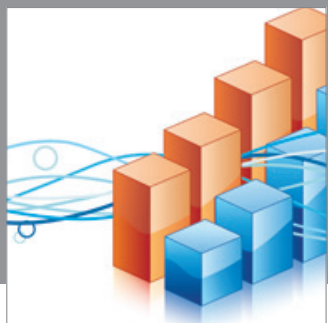

Advances in

Operations Research

mansans

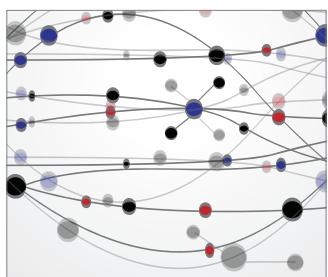

The Scientific World Journal
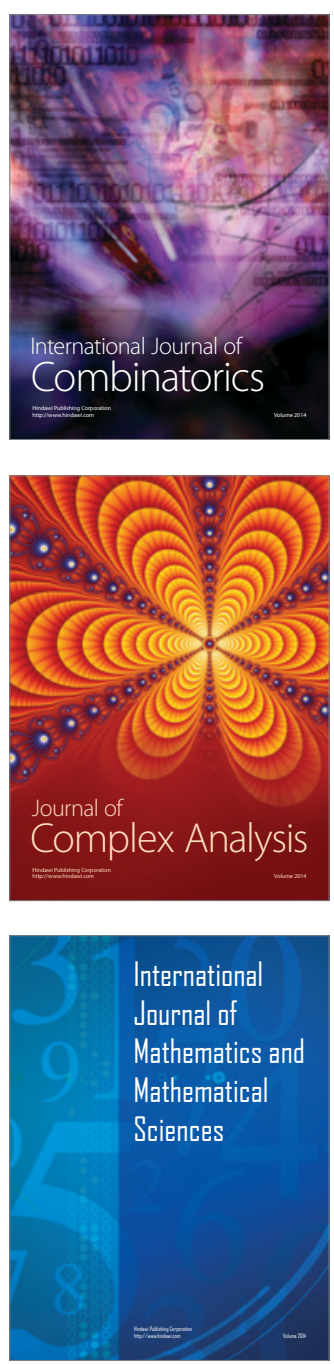
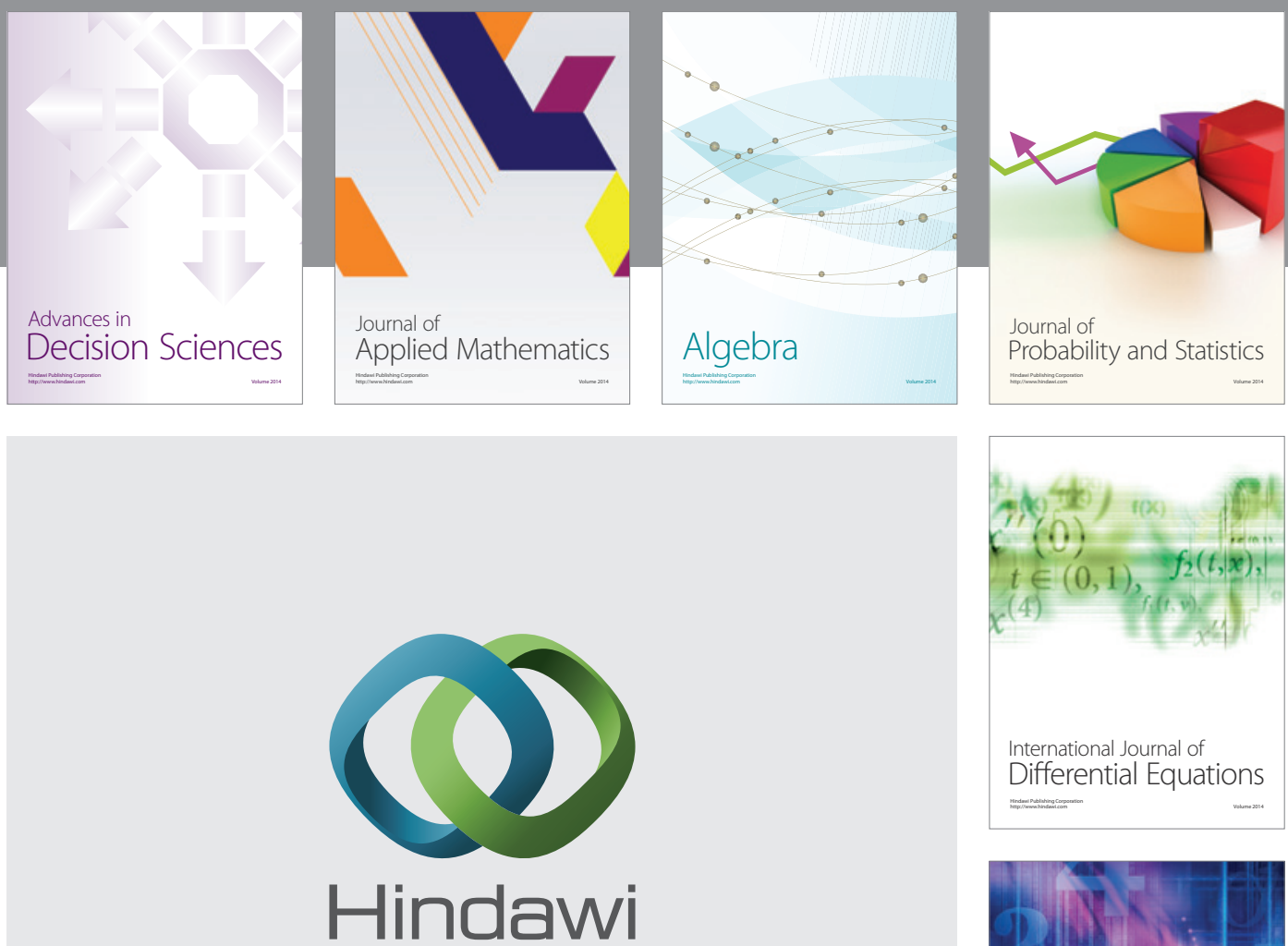

Submit your manuscripts at http://www.hindawi.com
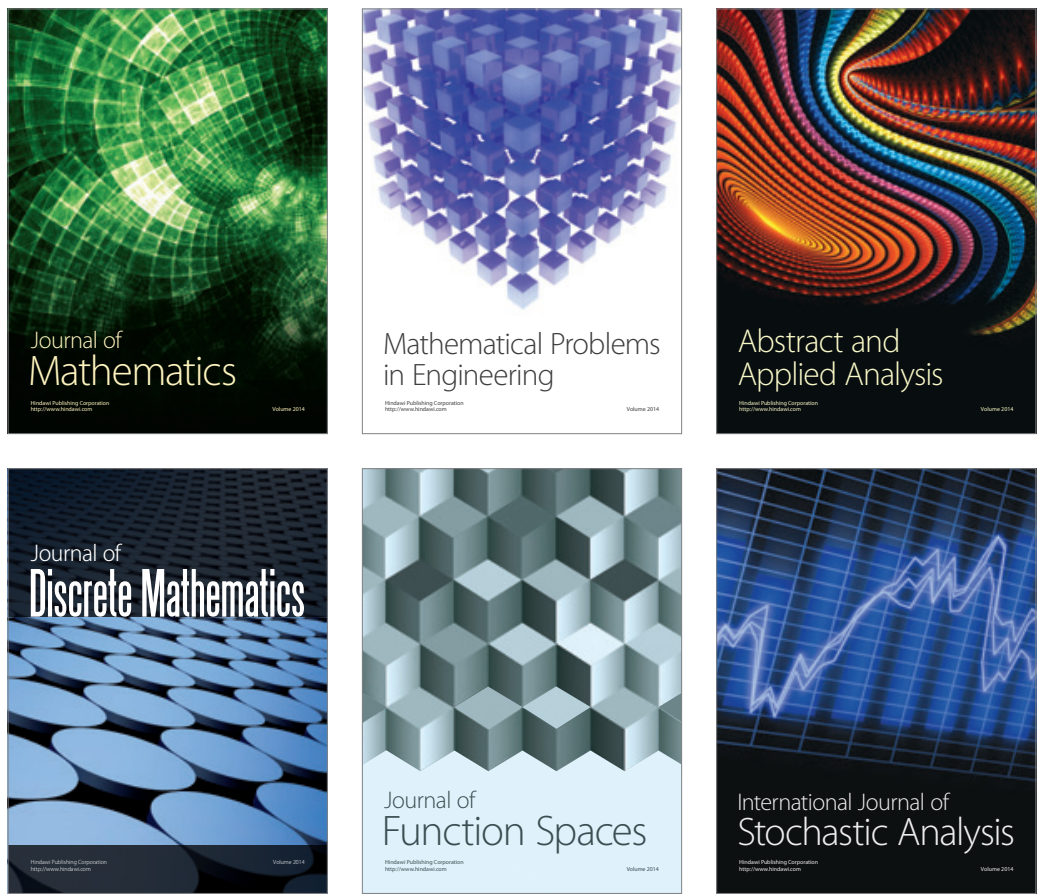

Journal of

Function Spaces

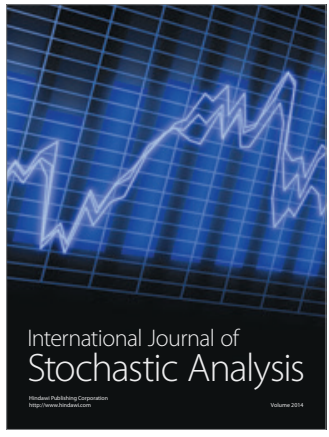

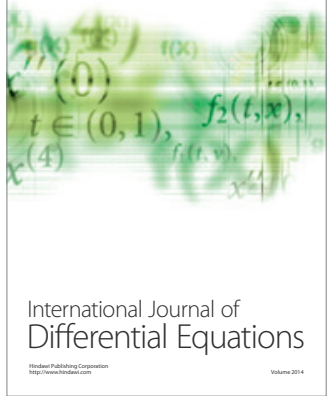
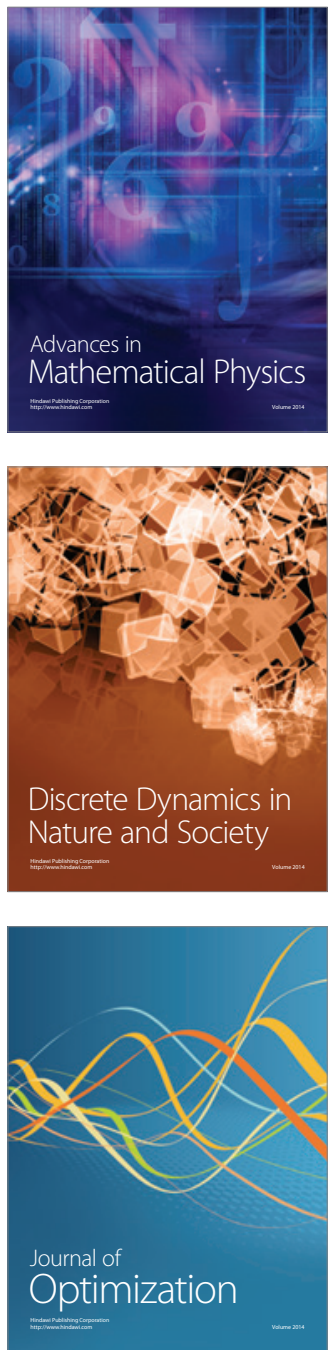